\title{
Costs and Effects of Microsurgery versus Radiosurgery in Treating Acoustic Neuroma
}

\author{
L. van Roijen ${ }^{1}$, H. G. T. Nijs ${ }^{1}$, C. J. J. Avezaat ${ }^{2}$, G. Karlsson ${ }^{3}$, C. Linquist ${ }^{4}$, K. H. Pauw ${ }^{5}$, and \\ F. F. H. Rutten
}

${ }^{1}$ Institute for Medical Technology Assessment, Erasmus University Rotterdam, The Netherlands, ${ }^{2}$ Department of Neurosurgery, University Hospital Rotterdam, The Netherlands, ${ }^{3}$ Stockholm School of Economics, Center for Health Economics, Sweden, ${ }^{4}$ Department of Neurosurgery, Karolinska Hospital, Stockholm, Sweden, and ${ }^{5}$ Department of Otorhinolaryngology, University Hospital Rotterdam, The Netherlands

\section{Summary}

This study analyses costs and effects of treating acoustic neuroma patients by using microsurgery compared to radiosurgery. Radiosurgery is the stereotactic application of radiotherapy and an innovative medical technology. Cost and effect estimates of conventional treatment were based on a retrospective study in the Netherlands. Similar data for a comparable group of patients in Sweden were collected for radiosurgery, as this treatment option is currently not available in the Netherlands.

Fifty-three acoustic neuroma patients who had been operated on the University Hospital Rotterdam between November 1990 and February 1995 were included. This group was compared with 92 acoustic neuroma patients treated with radiosurgery (Gamma Knife. Stockholm, Sweden) in the same period. Data on health care use were collected from patient files. To obtain data on production losses and quality of life, a questionnaire was sent by mail in February 1995. This booklet consisted of the Health and Labourquestionnaire (HLQ), the Short Form-36 (SF36) and the EuroQol. The response rate was $92 \%$.

Direct costs for microsurgery amounted to Dfl. $20.072,-$ and for radiosurgery to Dfl. $14.272,-$. Indirect costs were respectively Dfl. 16.400 ,- and Dfl. 1.020,- . General health rating was better for radiosurgery than for microsurgery. On the whole, differences in clinical outcomes between the two patient groups were small. Assuming a reasonable occupancy rate of the expensive radiosurgery equipment, we demonstrated that for the short term treating patients with acoustic neuroma with an extra-meatal tumour diameter of less than 3 centimeters, radiosurgery is more cost-effective than microsurgery.

Keywords: Acoustic neuroma; gamma knife radiosurgery; cost effectiveness.

\section{Introduction}

Radiosurgery comprises the stereotactic application of radiotherapy and is regarded as an innovative medical technology. It is a treatment method in which a small target volume in the patient's body is localised with great accuracy and subsequently irradiated using a single high dose of ionising radiation. Current indications for radiosurgery include, among others, arteriovenous malformations, brain metastases and benign skull base tumours (e.g., acoustic neuroma, meningiomas). Conventional surgery consists of craniotomy with surgical resection of the lesion. From the side of the Dutch government and the neurosurgeons there is a growing demand for cost-effectiveness analyses in order to evaluate these two treatment modalities.

Pollock et al. were the first to compare the results of microsurgery and radiosurgery [6]. Stereotactic radiosurgery proved to be an effective and less costly treatment of unilateral neuromas less than $3 \mathrm{~cm}$ in diameter. However, their study has some limitations. First, patients were treated with radiosurgery or microsurgery according to patients' preference, which reduces the comparability of the two groups. Second, the evaluation of costs was limited to length of hospital stay, management charges and effect on employment status. Third, only patient satisfaction was measured to capture the patients' perspective. In the present study we also have chosen acoustic neuroma as an example for an evaluation of radiosurgery. Consecutive series of eligible patients were studied. Cost and effect estimates of conventional treatment were based on a retrospective study in The Netherlands. Similar data for a comparable patient group in Sweden were collected for radiosurgery, as this treat- 
ment option is currently not available in The Netherlands. We calculated total direct- and indirect costs. Instead of patient satisfaction, we measured health related quality of life by standardised instruments. Health related quality of life constitutes the common denominator from the patients' perspective, when comparing two entirely different treatment modalities. Additionally, some relevant clinical outcomes are presented.

In short, we present a comparison of costs and effects of treating acoustic neuroma patients with microsurgery versus radiosurgery, as if radiosurgery was implemented in The Netherlands (hence: for costs, Swedish volumes are multiplied by Dutch prices). As all parameters and assumptions are stated explicitly, our study provides a framework for making similar comparisons for other countries or situations.

\section{Patients and Methods}

\section{Identification of Patients}

Radiosurgery refers to closed-skull single dose irradiation of an intracranial target with 1 millimeter precision. Since 1968, radiosurgery is performed using a Gamma Knife in the Karolinska Hospital in Stockholm. In the present paper, radiosurgery by using a Gamma Knife will be referred to as "radiosurgery". For comparison with conventional surgery, the tumour diameter should not exceed $30 \mathrm{~mm}$. Analysing conventional therapy we used data from the University Hospital Rotterdam. Costs and effects were studied retrospectively over a period of 4 years. This design was chosen because a prospective randomised study would take too long to complete. Fifty-three acoustic neuroma patients, who had been operated on the University Hospital Rotterdam between November 1990 and February 1995, were included. The Dutch patient group was compared with a group of 92 Swedish acoustic neuroma patients treated with radiosurgery during the same period with similar age and sex composition and comorbidity, who met the same medical criteria (unilateral tumour, extra meatal diameter less than $30 \mathrm{~mm}$, no prior treatment).

We analysed costs from the day of either micro- or radiosurgery. The pre-treatment period is not considered because no major differences in costs are to be expected for diagnostic procedures.

The total booklet contained a questionnaire for collecting data on production losses due to illness, the so called "Health and Labour" questionnaire (HLQ), general health status instruments and a standard set of socio-demographic questions on age, sex, education and employment status. Comorbidity was assessed by a standard list of 27 chronic conditions [1]. For both study groups patient questionnaires were sent by mail in February 1995, with reminders after two weeks. In case patients did not respond after the reminder, patients were approached by phone.

\section{Direct Costs}

Direct costs represent the costs as a consequence of surgical operation or radiosurgery session, hospital days, radiological procedures, laboratory tests, medication and outpatient visits (after treatment). In order to collect these data on health care use from patient files, registry-forms were developed, which were identical for Rotterdam and Stockholm.

For both treatments, the costs were calculated by multiplying Swedish and Dutch volumes by Dutch prices of 1995 (as if radiosurgery was implemented in The Netherlands). The average costs presented are based on the average follow-up time, which was similar for both groups. Estimating the costs for diagnostic procedures and laboratory tests, tariffs were used in case they reflected cost prices. Unit prices were calculated for the radiosurgery sessions, operation, hospital days and outpatient visits. Labour costs and capital cost for treatment with radiosurgery and the operation were calculated separately. Based on observations of the treatment process and the microsurgical operation, we estimated the intensity of different types of labour, in terms of fraction of the session/operation time. Estimating the capital costs we calculated annuities per year, using an interest rate of $5 \%$. A life span of 12 years was assumed for the radiosurgical equipment. For the modification of the building a life-span of 15 years was assumed, because the total life span of the building is estimated at 50 years while the University Hospital Rotterdam has existed for 35 years. Cobalt resources have to be replaced after 7 years. We allocated capital costs to cases assuming an average number of 200 patients per year (including all diagnoses) treated with radiosurgery. Based on the percentage of costs of non-medical staff, education and general hospital services in the total costs of the Department of Neurosurgery, "overhead" costs were calculated as $40 \%$ of the costs per session. Labour costs are based on the average gross earnings for employees in university hospitals for 1995. Due to important differences in resource use between normal- and intensive care hospital days, unit prices for both kinds of hospital days were calculated.

\section{Indirect Costs}

Indirect costs are the value of production losses because patients are unable to perform paid or unpaid work. For the valuation of production losses of paid labour we used the average production value per working person for the Netherlands [5]. Studies indicate that a reduction of annual labour time causes less than proportional decrease in labour productivity per year $[2,4,12] \mathrm{We}$ assumed this elasticity to be 0.8 .

In order to collect data on production loss between the two treatment groups the Health and Labour-questionnaire (HLQ) was applied [8]. The HLQ enables one to estimate production losses of paid and unpaid labour and the ability to work as an indicator of health status. The complete HLQ consists of four modules (absence from work, reduced productivity at work, unpaid production and experience of trouble with paid and unpaid work) [7]. In view of this study population we applied the first, third and fourth module. According to module 1 respondents with paid jobs were asked to mark on a bar for each (half) day(s) of the two weeks preceding the assessment, whether they performed their work or were absent due to health problems or for other reasons (e.g., weekend, off days). Module 3 concerned unpaid production like household work, shopping, care for children and jobs around the house (like gardening). We asked whether household tasks normally done by the patient were taken over by other family members, neighbours and/or paid help. In module 4 of the HLQ subjects, having a paid job, were requested to indicate the impediments experienced while performing their job. The response categories are as follows: "no impediment", "some impediment" and "a lot of impediment". The item 
scores are 0,1 and 2 respectively. So, the impediment score ranges from 0 to 2 . Consequently, a higher score means more impediment.

\section{Health Status Instruments}

We assessed health related quality of life by using non-disease specific validated questionnaires. These instruments measure basic values (physical, psychological and social functioning) which are relevant for everyone's health status [9]. A combination of two generic questionnaires, The Medical Outcomes Study 36-item Short Form-36 (SF36) and the EuroQol (descriptive instrument and the thermometer) were applied to investigate whether differences between patients treated by radiosurgery and conventional surgery were consistent if measured by different instruments $[3,9]$. The SF36 consists of 36 items assigned to conceptual domains of Physical Functioning, Social functioning, Role limitations, Mental Health, Vitality, Pain, General Health Perceptions and Health Change. The EuroQol classification consists of five items (Mobility, Self-care, Usual activities, Pain/discomfort and Anxiety/depression), each following the general form: no problems - some problems - extreme problems. In addition, evaluation of perceived health is assessed with a visual analogue scale ranging from 0 (worst imaginable health state) to 100 (best imaginable health state).

\section{Statistics}

Standard statistical techniques were used. Multiple linear regression analyses were applied to compare quality of life outcomes, corrected for age, sex, comorbidity and educational level.

\section{Results}

Response

Fifty three questionnaires were mailed to the patients treated by microsurgery; 49 patients (92\%) responded. Ninety-two patients treated by radiosurgery were requested to participate in the study. In Sweden the overall response rate was also $92 \%$ (90 patients).

\section{Patient Characteristics}

In Table 1 patient characteristics are shown. Characteristics reflect the patient's status at study entry (follow-up time for both groups is similar). In the radiosurgery group educational level is higher than in the microsurgery group (median level 4 versus 3, $\mathrm{p}<0.001$ ). Yet, some caution is warranted in comparing school systems in two countries. Total number of reported comorbidities from a standard list of 27 most prevalent diseases and illnesses was not different. However, the radiosurgery group reported more "serious heart disease or myocardial infarction" (14\% versus $2 \%$ for microsurgery $p<0.02$ ) and "arthritis (reuma) of hands or feet" ( $17 \%$ versus $4 \%$ for microsurgery $\mathrm{p}<0.01)$.
Table 1. Patient Characteristics at Study Entry

\begin{tabular}{lll}
\hline & Microsurgery & Radiosurgery \\
\hline $\mathrm{n}$ & 53 & 92 \\
$\begin{array}{l}\text { Follow-up; months } \\
\text { [median, min-max] }\end{array}$ & $24(1-53)$ & $24(0.5-75)$ \\
Age; yr (mean; SD) & $52(11)$ & \\
Sex; \% Male & $51 \%$ & $55(14)$ \\
Tumour diameter & & $34 \%$ \\
$\quad<15$ mm & $52 \%$ & \\
$\quad 15-30$ mm & $48 \%$ & $53 \%$ \\
Comorbidity & & $47 \%$ \\
$\quad 0-1$ & $51 \%$ & \\
$2-4$ & $43 \%$ & $57 \%$ \\
$\quad>5$ & $6 \%$ & $13 \%$ \\
Educational level ${ }^{\text {a, }}$ median & 3 & 4 \\
Paid work (\%) & $53 \%$ & $47 \%$ \\
\hline
\end{tabular}

aRange of possible values: $1-7$

\section{Direct Costs}

Table 2 presents the average number and costs of the most relevant medical procedures per patient. According to anaesthesia reports, the average duration of the micro-surgical operation was 7 hours and 24 minutes. Given this duration and labour intensiveness, the average costs of the operation were over Dfl. $7.700,-$. In case of treatment with radiosurgery the average duration was 7 hours, of which over 5 hours was required for application of the stereotactic frame and to carry out MRI and dose planning. Table 2 shows the intensiveness of different types of labour in terms of their fraction of total treatment time.

The investment costs of the radiosurgery equipment amounted to Dfl. 6.7 million, modification of the building Dfl. 3 million and the replacement of the Cobalt resources almost Dfl. 700.000. The annual costs per patient are based on 200 treatments per year, which may be considered as the base scenario. For radiosurgery nearly $70 \%$ of the direct costs were attributed to the costs of the session with the Gamma knife.

The average length of total hospital stay from the day of surgery of patients treated by microsurgery is 13 days, nearly 11 days of normal care and over 2 days of intensive care. $65 \%$ of the patients treated by radiosurgery required hospitalisation. The total length of stay varied from 1 to 5 days. The total average length of stay was 1.6 days, 1.1 days before treatment and 0.5 days after treatment. The costs of a normal 
Table 2. Average Number of the Most Important Procedures and Costs (in Dutch Guilders, 1995) per Acoustic Neuroma Patient from Day of Operation or Treatment Radiosurgery

\begin{tabular}{|c|c|c|c|c|}
\hline & \multicolumn{2}{|c|}{ Microsurgery } & \multicolumn{2}{|c|}{ Radiosurgery } \\
\hline & Number & Costs & Number & Costs \\
\hline \multicolumn{5}{|c|}{ Hospitalisation (duration in days) } \\
\hline normal care & 10.5 & 5.554 & 0.5 & 265 \\
\hline intensive care & 2.4 & 3.387 & & \\
\hline \multicolumn{5}{|l|}{ Scans } \\
\hline MRI-scan & 0.3 & 275 & 1.5 & 1.683 \\
\hline CT-scan & 1.2 & 376 & 2.0 & 705 \\
\hline \multicolumn{5}{|c|}{ Microsurgical operation/treatment } \\
\hline Capital costs ${ }^{\mathrm{a}}$ & & & & 5.652 \\
\hline Labour costs ${ }^{b}$ & & 2.857 & & 1.378 \\
\hline Overhead & & 4.860 & & 2.812 \\
\hline Outpatient visits & 3.9 & 712 & 0.4 & 728 \\
\hline Other $^{d}$ & & 2.052 & & 1.050 \\
\hline Total Costs & & 20.072 & & 14.272 \\
\hline
\end{tabular}

\begin{tabular}{|c|c|c|}
\hline (in fraction of total operation/treatment time) & Microsurgery & Radiosurgery \\
\hline neurosurgeon & 1 & 1 \\
\hline neurosurgeon ass. & 1 & - \\
\hline anaesthetist & 0.5 & - \\
\hline anaesthesia nurse & 1 & - \\
\hline nurse & 2 & 1.5 \\
\hline ENT physician & 0.5 & - \\
\hline physicist & - & 0.4 \\
\hline radiologist & - & 0.2 \\
\hline
\end{tabular}

'With respect to microsurgery 'overhead' consists of costs of the operation room and indirect costs. For radiosurgery, 'overhead' (non-medical staff, education and general hospital services) is $40 \%$ of the costs per session.

${ }^{\mathrm{d}}$ Including costs of visits to eye specialist and ENT physician, medication and laboratory tests.

hospital day in the neurosurgical department amounted to Dfl. 530,- per day, and to Dfl. 1426,- for intensive care. So, the mean costs for hospitalisation from the day of the treatment were nearly Dfl. $9.000,-$ per patient treated by microsurgery, compared to only Df1. 265,- for radiosurgery (Table 2).

The number of post-operative MRI- and CT-scans were on average higher in the Gamma Knife group compared to the patients treated with microsurgery, see Table 2 .

The total costs of outpatient visits were over Dfl $700,-$ for the microsurgery group. We assumed on average 4 post-treatment outpatient visits for the Gamma Knife group.

In the Dutch patient group the average costs of laboratory tests amounted to Dfl. 900,- after the operation.

\section{Indirect Costs}

The Dutch patients with paid jobs were absent from work for 3 months after surgery. Patients treated by radiosurgery were on average absent from work for one working week after treatment. The average indirect costs per patient with paid work in the microsurgery group amounted to Dfl. $16.400,-$. In the group who underwent radiosurgery these costs were only Dfl. 1020,--

Four of the patients in the surgical group recorded that they were not able to return to their job due to consequences related to the operation. This equals $17 \%$ of patients with a paid job in this treatment group. Only one person in the radiosurgery group reported he was incapacitated due to the consequences of the treatment, which equals $2 \%$ of the study population with paid work. 
Table 3. Average Number of Days and Costs (in Dutch Guilders, 1995) Due to Absence from Work, Number of Incapacitated Persons and Impediment Score for Paid Work Due to Treatment of Acoustic Neuroma

\begin{tabular}{lll}
\hline & Microsurgery & Radiosurgery \\
\hline $\begin{array}{l}\text { Number of days } \\
\text { absence from work }\end{array}$ & 60 & 5 \\
$\begin{array}{l}\text { Indirect costs due to } \\
\text { absence from work (in Dfl.) }\end{array}$ & 16.400 & 1.020 \\
$\begin{array}{l}\text { Number of incapacitated patients } \\
\text { (in \% of paid workers) }\end{array}$ & $4(17 \%)$ & $1 .(2 \%)$ \\
Impediment score (mean,SD) & $0.55(0.65)$ & $0.11(0.32)$ \\
\hline
\end{tabular}

Table 4. Patients' Performance of Household Production, Due to Treatment of Acoustic Neuroma

\begin{tabular}{lll}
\hline & $\begin{array}{l}\text { No. of patients (\%) } \\
\text { Microsurgery }\end{array}$ & $\begin{array}{l}\text { No. of patients (\%) } \\
\text { Radiosurgery }\end{array}$ \\
\hline Self-performing & $73 \%$ & $87 \%$ \\
Family members & $21 \%$ & $10 \%$ \\
Others (e.g. neighbours) & $3 \%$ & $1 \%$ \\
Alpha & $3 \%$ & $1 \%$ \\
Home-help & $5 \%$ & $1 \%$ \\
Other paid help & $5 \%$ & $0 \%$ \\
\hline
\end{tabular}

For individuals with paid jobs the average impediment score was 0.55 for the group receiving surgery, and 0.11 for those treated with radiosurgery.

We asked whether household tasks, usually performed by the respondent, were postponed or taken over by other members of the household, family or friends and/or paid workers. In the patient group treated with microsurgery $27 \%$ indicated that tasks were taken over, mainly by family members, compared to $13 \%$ in the group treated with radiosurgery, see Table 4.

\section{Total Costs}

Total costs (direct and indirect) for microsurgery amounted to Dfl. 36.472,- and for radiosurgery to Dfl. $14.272,-$. The fact that total costs for microsurgery were over two times higher than for radiosurgery, was mainly due to the difference in the number of days of hospitalisation and the substantial discrepancy in indirect costs. The direct costs dominate in both treatments, but to a much larger extent for microsurgery.

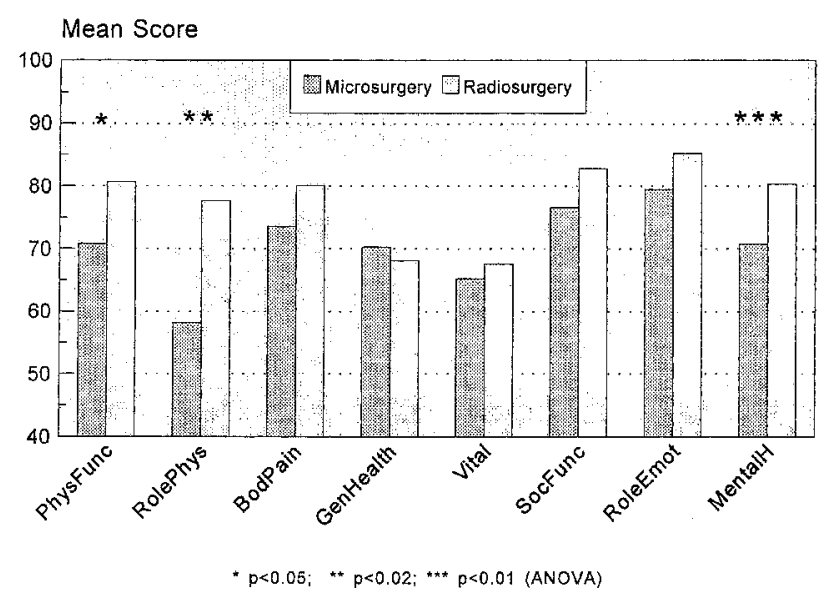

Fig. 1. Quality of life (SF36). Health related quality of life after microsurgery and radiosurgery

\section{Health Related Quality of Life}

General health rating was better for radiosurgery (excellent $16 \%$, (very) good $65 \%$, poor or fair 19\%) than for microsurgery (excellent $4 \%$, (very) good $65 \%$, poor or fair $30 \%, \mathrm{p}<0.01)$.

In Fig. 1 SF36-scores are presented. For 5 out of 8 domains no significant differences were found. The radiosurgery group had significantly higher scores, indicating better health related quality of life, than the microsurgery group for the domains of physical functioning (e.g., walking, climbing, vigorous activities), role physical (e.g., performing work/activities), and mental health (happiness, nervousness, mood). Though statistically significant, the differences were moderate: within 0.5 SD difference.

Also when measured with the EuroQol, the radiosurgery group scored higher than the microsurgery group. Mean (SD) weighted EuroQol-score 0.89 $(0.15)$ for radiosurgery versus $0.77(0.18 ; p<0.01)$ for microsurgery, also a difference around $0.5 \mathrm{SD}$.

These results remain present when analysing these data per period, with the categories <1 year, 1-2 years, and 2-4 years past treatment. No trend in time was found for either group. All differences remained significant and of similar magnitudes when corrected for age, sex, comorbidity and educational level.

Regardless of the instrument used, the radiosurgery group had significantly higher health related quality of life than the microsurgery group.

\section{Clinical Outcomes}

In Table 5 some relevant short and long term clinical outcomes are shown. Short term outcomes in the microsurgery group reflect the typically surgery-asso- 


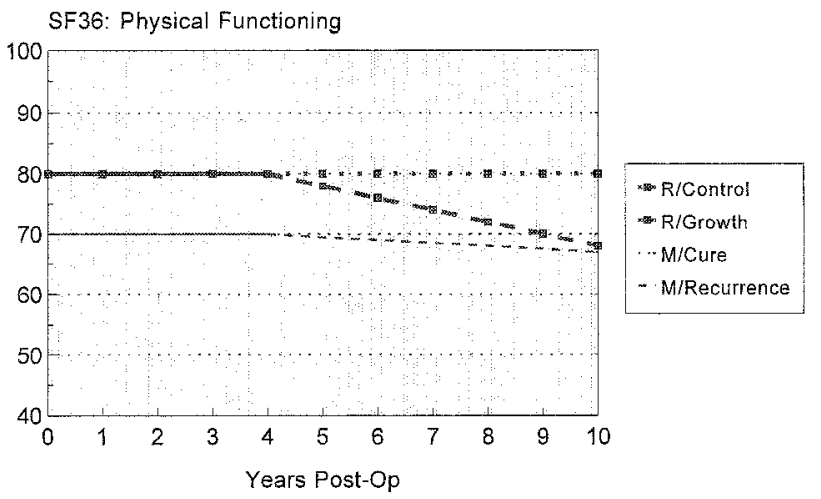

Fig. 2. Quality of life: conceptual courses. Conceptual time profiles of health related quality of life up to 10 years after micro $(M)$ - or radiosurgery $(R)$

Table 5. Clinical Outcomes

\begin{tabular}{lcl}
\hline & Microsurgery & Radiosurgery \\
\hline Short term outcomes & & \\
Meningitis & $6.5 \%$ & $0 \%$ \\
Hydrocephalus & $7.0 \%$ & $0 \%$ \\
Liquor cyst & $4.8 \%$ & $0 \%$ \\
Urinary infection & $3.0 \%$ & $0 \%$ \\
Respiratory infection & $2.0 \%$ & $0 \%$ \\
Long term outcomes & & \\
N. VII; \% affected & $10 \%$ & $2 \%$ \\
N. V; \% affected & $0 \%$ & $0 \%$ \\
\hline
\end{tabular}

ciated complications, such as meningitis and hospitalassociated complications, e.g. urinary and respiratory tract infection. These were absent in case of radiosurgery. Facial- and trigeminal nerve function were dichotomised in "affected" or "not-affected". Hydrocephalus and liquor cyst where scored as a complication only in case of post-operative repair.

On the whole, long term clinical outcomes between the two patient groups were comparable. However, facial nerve paralysis may be less frequent after radiosurgery than after microsurgery.

\section{Sensitivity Analyses Direct Cost Estimates}

In the base scenario we assumed 200 treatments per year for radiosurgery. Table 6 shows the direct costs per patient treated by radiosurgery at different occupancy rates. As in the base scenario we assumed an interest rate of 5\%, a life span of 12 years for the radiosurgical equipment, and of 15 years for modifying the building, and a replacement of Cobalt resources after 7 years.
Table 6. Capital Costs per Patient for Treatment with Radiosurgery Assuming Different Occupancy Rates (in Dutch Guilders, 1995)

\begin{tabular}{lrrrr}
\hline $\begin{array}{l}\text { Number of treatments } \\
\text { per year }\end{array}$ & 100 & 200 & 300 & 400 \\
\hline Radiosurgical equipment & 7.542 & 3.771 & 2.514 & 1.885 \\
Cobalt sources & 1.062 & 531 & 354 & 266 \\
Building modification & 2.700 & 1.350 & 900 & 675 \\
Total capital costs & 11.304 & 5.652 & 3.768 & 2.826 \\
\hline
\end{tabular}

A scenario of 100 treatments per year, including all diagnoses, would produce an average of Dfl. 21.850,per patient, which is slightly above the level of the costs for microsurgery. Total direct costs would decline to Dfl. 11.746,-- per treatment if 300 treatments per year are assumed. The total average direct costs would be reduced by nearly Dfl. $10.000,-$ per patient if the occupency rate would be increased to 400 treatments per year. As may be expected, substantial economics of scale can be demonstrated.

\section{Discussion}

Assuming a reasonable occupancy rate of the expensive radiosurgery equipment, we demonstrated that for the short term (up to 4 years) treating patients with an extra-meatal tumour diameter of an acoustic neuroma less than 3 centimeters with radiosurgery was more cost-effective than microsurgery.

The goal of microsurgery is radical tumour removal and the goal of radiosurgery is arrest of tumour growth. A strictly clinical comparison, e.g. post-operative tumour diameter, is therefore not relevant, since microsurgery would be more favourable by definition. The study of Pollock indicates that radiosurgery can achieve long-term growth control and may, therefore, be considered as at least equivalent to cure [6]. The follow-up period of our study is too short to allow a definite conclusion regarding the efficacy of the radiosurgical treatment. Moreover, since 1988 the radiation dose of the radiosurgical unit at the Karolinska Hospital has been decreased in order to reduce morbidity, but to study the effect on long-term tumour control a longer follow-up period is needed in view of the slowly progressive natural growth of the tumour.

From the patients' point of view also the shortterm health related quality of life is important (especially in older patients). Figure 2 shows several conceptual time profiles of health related quality of life 
up to 10 years after both micro- or radiosurgery. According to Noren et al. radiosurgery will succeed in tumour control. So, health related quality of life is expected to remain constant. However, in a subsample of patients treated with radiosurgery, tumour control may not be achieved, indicated in the figure as tumour growth: this will presumably lead to a decrease of health related quality of life. For the short term up to 4 years, health related quality of life of patients is on average lower for operated patients compared to patients treated with radiosurgery. Study indicated that tumour recurrence after complete resection will be between $0 \%$ and $9 \%$ [6]. In case of tumour recurrence, health related quality of life is expected to decrease in time (see Fig. 2).

Other studies support the reproducibility and the comparability of the scale scores of the SF36 in Sweden, the United Kingdom and the United States. The pattern of predictive relationships between the SF36 scales and the General Health Scale was consistent across these three countries and demonstrated the importance of the SF36 health concepts in explaining variations in personal health evaluations. Findings to date from The Netherlands indicate it is the same for the Dutch version [10] of the SF36.

The average costs per patient treated by radiosurgery depend to a large extent on the number of treatments per year. In our base scenario the total number of treatments was 200 per year. According to a report of the Dutch Health Council, accepted indications for radiosurgery include acoustic neuroma and arteriovenous malformations [11]. So, it is reasonable to assume that the number of treatments will exceed the base estimate of 200 per year, also in case a single center for radiosurgical treatment for The Netherlands would be erected.

Absence from work and incapacity for work are influenced by the social security system of a country [8]. This study indicated that the highest level for absence from work rates occur in countries with more liberal criteria and procedures for entitlement. There are no major differences in the social security systems in case of absence from work between the two countries. However, with respect to incapacity for work the social security systems differ between the Netherlands and Sweden. Both the level and the length of the period of receiving incapacity for work benefits are relatively attractive in the Netherlands. As a consequence, an incapacity for work benefit is often preferred to an unemployment benefit. The Swedish gov- ernment pursues an active policy against incapacity for work, towards preventive arrangements as substitutes. So, this difference in the social security systems may have increased the number of incapacitated in the Dutch patient group compared to the Swedish.

\section{Acknowledgements}

We would like to thank Annette de Jong and Maarten Driesen for collecting data. Joseph McDonnell is gratefully acknowledged for his support in completing statistical analyses. This study was financially supported by Elektra Instrument $A B$.

\section{References}

1. CBS (Netherlands Central Bureau of Statistics) (1991) National Health Survey 1989 and 1990. Den Haag

2. CPB (Central Planning Bureau) (1987) Uncertainties on labour time reduction in the medium term (in Dutch), working document 14, Den Haag

3. Essink-Bot ML, Stouthart MEA, Bonsel GJ (1993) Generalizability of valuations on health status collected with the EuroQol-Questionnaire. In: Health Economics, Vol 2, pp 237-246

4. Koning J, Tuyl FAWM (1984) The relation between labour time, production and employment (in Dutch). Netherlands Economic Institute, Rotterdam

5. Koopmanschap MA, Rutten FFH, Ineveld BM van, Roijen $L$ $\operatorname{van}(1995)$ The friction cost method for estimating the indirect costs of disease. J Health Econ 14: 171-189

6. Pollock BE, Lunsford LD, Kondziolka D, Flickinger JC, Bissonette DJ, Kelsey SF, Jannette PJ (1995) Outcome analysis of acoustic neurinoma managements: a comparison of microsurgery and stereotactic radiosurgery. Neurosurgery 36 : 215-223

7. Roijen L van, Essink-Bot ML, Koopmanschap MA, Bonsel G, Rutten FFH (1996) Labour and health status in economic evaluation of health care. Int J Technol Assess Health Care 12 (3): 404-415

8. Roijen L van, Koopmanschap MA, Rutten FFH, Maas Pj van der (1995) Indirect costs of disease: an international comparison. Health Policy 33: 15-29

9. Ware JE, Sherbourne CD (1992) The MOS 36-item ShortForm Health Survey (SF-36). I. Conceptual framework and item selection. Med Care 30: 473-483

10. Ware JE, Keller SD, Gandek B, Brazier JE, Sullivan M (1995) Evaluating translations of health status questionnaires. Int $\mathrm{J}$ Technol Assess Health Care 11: 525-551

11. National Health Counsil (1994) Stereotactic radiation; Gamma Knife and other techniques (in Dutch). The Hague, 14 October 1994

12. WRR (Scientific Council for Government Affairs) (1977) Do we make it work? Research of the relation between active and inactive people (in Dutch), Den Haag

Correspondence: L. van Roijen, Institute for Medical Technology Assessment, Erasmus University Rotterdam, P. O. Box 1738, NL-3000 DR Rotterdam, The Netherlands. 